\title{
Scale effects in numerical modelling of beach profile erosion
}

\author{
Filipa S. B. F. Oliveira $\uparrow$ and Joana Contente $\uparrow$
}

†Laboratório Nacional de Engenharia

Civil, Av. do Brasil, 1700-066 Lisboa,

Portugal

f.oliveira@lnec.pt

joana.contente22@gmail.com

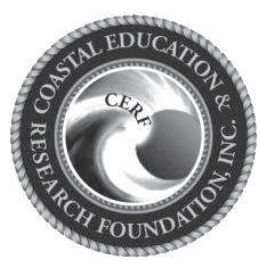

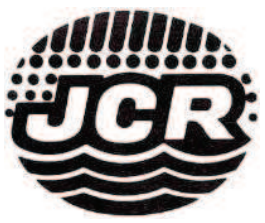

www.JCRonline.org

ABSTRACT

Oliveira, F.S.B.F. and Contente, J., 2013. Scale effects in numerical modelling of beach profile erosion. In: Conley, D.C., Masselink, G., Russell, P.E. and O'Hare, T.J. (eds.), Proceedings $12^{\text {th }}$ International Coastal Symposium (Plymouth, England), Journal of Coastal Research, Special Issue No. 65, pp. 1815-1820, ISSN 0749-0208.

The beach profile morphodynamic processes can be relatively well reproduced in large scale physical models. However, the cost associated to their use makes this methodology not always practicable. As alternative, it is desirable to explore and apply smaller scales laboratory infrastructures with confidence, despite the different scaling laws, that is, the impossibility to simulate all relevant variables in correct relationship to each other. The combination of physical and numerical modelling can be a methodology to improve the required confidence (HYDRALAB III European Programme). Scale effects in a numerical process-based beach profile model, applied to simulate beach profile evolution under erosive wave conditions, were investigated in the present study. The numerical model was applied at laboratory and prototype scales, first with the default settings and then calibrated. Its performance was evaluated using the Brier Skill Score. The numerical results of the profile evolution in both scales were compared after reducing the results from the prototype scale to the laboratory scale. The profile erosion prediction was overestimated in the laboratory scale and underestimated in the prototype scale. Despite the calibration parameter that causes the largest modifications of the beach profile being the same in both scales, the Gamma2 wave parameter, the most efficient parameter was different. For most of the calibration parameters, the value for the best case was not coincident in both scales. It was concluded that there are scale effects using the Litprof model.

ADDITIONAL INDEX WORDS: Coastal erosion, morphodynamic numerical modelling, short-term, laboratory-numerical experiment, downscaling nearshore and beach processes.

\section{INTRODUCTION}

The coastal morphodynamic processes can be relatively well reproduced in large scale physical models. However, the cost and time associated to their use make this methodology not always the most practicable one. Alternatively, small-scale physical models have several limitations in reproducing the sediment dynamics as one cannot satisfy all different scaling laws. On the other hand, morphodynamic numerical models can be less expensive and more flexible, but, despite the improvement achieved in the last decades, limitations still remain, particularly in what concerns the swash zone dynamics and the onshore sediment transport associated with beach recovery after erosion events. Thus, the combination of both approaches, in an integrated and balanced way, when exploring their individual strengths, can improve the simulation capacity of the complex processes involved in coastal problems (Frostick et al., 2011). Furthermore, this combined modelling can improve each methodology individually: on one hand, the parameters measurement in the laboratory, in different stages of the experiment, allows to execute a rigorous control of the numerical results and, thus, obtain greater confidence in the final predictions; on the other hand, the numerical simulation of laboratory conditions allows testing a wider range of conditions, which not only would take more time but could also be impossible to test, due to reasons like infrastructure size limitation and financial cost.

Due to the high interest on the application of numerical models

DOI: 10.2112/SI65-307.1 received 07 December 2012; accepted 06 March 2013

(c) Coastal Education \& Research Foundation 2013 of short-term beach morphodynamics at the scale of the reduced scale laboratory models, it is necessary their validation at those smaller scales. Meaning that, it is required to understand if in order to perform combined modelling of beach profile evolution, one should perform the numerical modelling at the prototype scale and then reduce the results to the laboratory scale or perform the numerical modelling at the reduced scale directly. The objective of the present study was to answer the above question. For that, a beach profile evolution under erosive wave conditions case, previously reproduced in the laboratory, was simulated numerically at both scales, the laboratory and the prototype scales. After reduced to the laboratory scale, the numerical results at the prototype scale were compared with the numerical results at the laboratory scale. Thus, it was possible to investigate on the scale effects in a numerical process-based beach profile model, the Litprof model, for a case of beach profile evolution under erosive wave conditions.

\section{METHODOLOGY}

\section{Methodological Approach}

Aiming to evaluate the scale effects in numerical modelling of a beach profile erosion case it was implemented the following methodological procedure:

1. Numerical simulation of the case study at the laboratory scale.

1.1 Application of the model with the default parameters.

1.2 Model calibration.

2. Numerical simulation of the case study at the prototype scale. 
2.1 Application of the model with the default parameters.

2.2 Model calibration.

3. Comparison of the numerical results obtained at the laboratory and prototype scales, after reducing the prototype scale results to the laboratory scale results.

The application of this type of process-based morphodynamic models requires the knowledge of a high number of parameters associated to conditions of wave, flow, transport, morphodynamics, boundary and numerical stability, which values to use should correspond to the local physical conditions. Frequently, in engineering projects, it is not feasible the direct measurement of these parameters. Under such conditions, the procedure frequently used is either to apply values used in case studies with similar physical conditions reported in the literature, or to apply the default parameters, recommended by the model authors, which were defined as being the ones which best represent the widest range of possible physical conditions previously tested.

In this study, in both phases of the numerical model application (at the laboratory scale and at the prototype scale), it was performed a prior application of the model with the default parameters. The main calibration parameters of the model Litprof (DHI, 2008), which will be described in the last section of this chapter, are identified in Table 1, where the default values applied can also be seen. The calibration process of the model in both phases consisted in the testing different values of the calibration parameters in order to improve the similarity between the numerical and the experimental results. The calibration parameters were tested one by one, keeping the others constant with the default values. The values tested can also be seen in Table 1 .

\section{Case Study}

The conditions of hydrodynamic, morphology and sedimentology of the Buarcos beach, in Figueira da Foz, a littoral zone located in the west central coast of Portugal, previously studied and characterized (Oliveira, 2002; Larangeiro et al., 2003; Freire et al., 2004), were tested through physical modelling. Part of the laboratory tests performed, described in detail in Freire et al. (2008), namely the one correspondent the present case study, were executed in the COI2 infrastructure of LNEC, an irregular wave flume with dimensions $73 \mathrm{~m} \times 3 \mathrm{~m} \times 2 \mathrm{~m}$ (length, width and height) (Figure 1).

The present case study is a beach profile with 1:20 slope and sediment median diameter $D_{50}=0.4 \mathrm{~mm}$, performed at a scale $1: 6$ reduced model. The top of the beach profile was limited by a longshore coastal defence, which, due to the water level considered for this case study, $1.0 \mathrm{~m}$, was not submitted to direct wave action, that is, wave-structure interaction did not occur for this case study. The incident wave conditions were a JONSWAP spectrum with $H_{s}=0.37 \mathrm{~m}, T_{p}=3.27 \mathrm{~s}$ and 12 hours duration, where $H_{s}$ is the significant wave height and $T_{p}$ is the peak period. For the numerical simulation, the initial profile considered was the one obtained after the first hour, being, therefore, the total period of simulation 11 hours.

Under the erosive wave conditions the laboratory beach profile

Table 1. Calibration parameters tested

\begin{tabular}{lll}
\hline \hline Parameter $^{1}$ [unit] & Default values & Other tested values \\
\hline Gamma1 [-] & 0.88 & $0.6,0.7,0.8,0.9,1$ \\
Gamma2 [-] & 0.6 & $0,0.4,0.8,1$ \\
Beta [-] & 0.15 & $0.1,0.2$ \\
Max Angle $\left[{ }^{\circ}\right]$ & 30 & $10,15,20$ \\
Scale Parameter [-] & 1 & $0.8,1.2$ \\
\hline
\end{tabular}

${ }^{1}$ named according to the variables notation in the model interface

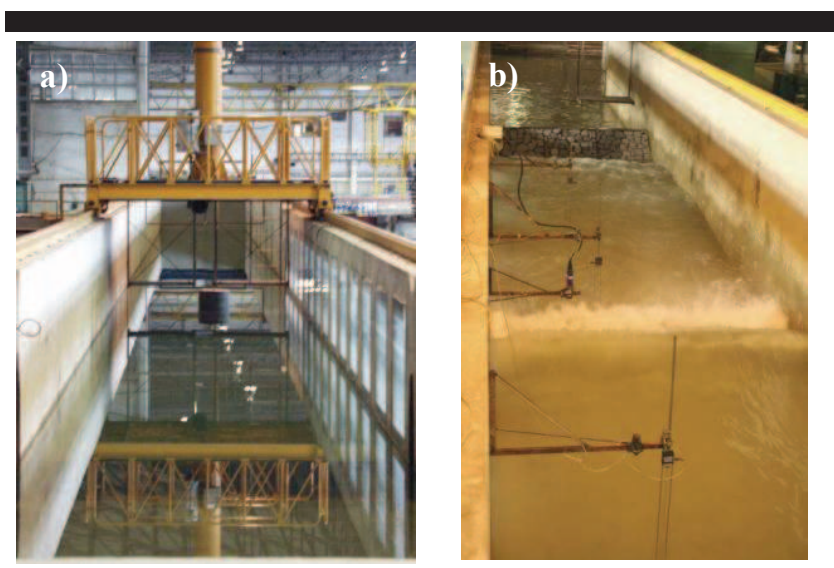

Figure 1. Experimental infrastructure (irregular wave flume COI2 of LNEC): a) general view of the flume and b) wave gauges deployed along the flume.

evolved from an initial planar profile to a typical bar-trough type of profile, were the trough was not deep but tended to be a platform (Figure 2). The still water level corresponds to the vertical coordinate, $z$, equal to zero. It should also be pointed out that during the experiment sediment escaped from the lower part of the profile to the flume (out of the monitoring zone).

For the laboratory reduced model the following conditions were considered for the downscaling (Freire et al., 2008):

- In the case of the hydrodynamic processes: a) the model was geometrically undistorted, that is, it was applied a unique length scale for all the variables $\left(n_{H}=n_{L}=n_{h}\right.$, where $\mathrm{n}$ is the prototype model relationship and the indexes $H, L$ and $h$ are the wave height, the wave length and the depth); b) the Froude number $F r=U /(g h)^{1 / 2}$, where $U$ is the characteristic flow velocity and $g$ is the gravitational acceleration) was considered similar in the prototype and the model; and c) the scaling laws of velocity, $U$, and time, $T$, resulted in $n_{U}=n_{T}=\left(n_{L}\right)^{0.5}=\left(n_{h}\right)^{0.5}$

- In the case of the sedimentary processes it was applied the suspended-load scaling law $n_{D 50}=\left(n_{h}\right)^{0.56}$, where $n_{D 50}$ and $n_{h}$ are the scaling relationship of the sediment median diameter and of the depth, respectively. An analysis of the sediment mobility number was performed in order to verify that the scaled model tests fell in the same sediment transport/bed regime as in the prototype.

\section{Numerical Model and Evaluation Criterion}

The numerical model applied for modelling the morphodynamics due to the wave induced currents was the Litprof model (DHI, 2008). It is a quasi-3D morphodynamic model, based

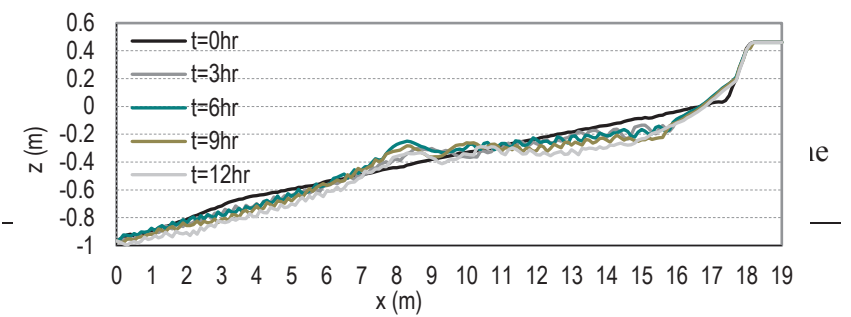


on the physical costal processes predominant in quasi-uniforme beaches, that is, beaches where the incident wave direction can be variable but the isolines of bathymetry are approximately parallel to the coastline (meaning that the gradients of the hydrodynamic and sedimentologic conditions alongshore are negligible).

The model describes the morphological modifications occurred in a cross-shore beach profile when submitted to a wave climate and sea level (tide and surge) time series. It is a numerical model composed by several sub-models of costal processes: an hydrodynamic model, a quasi-3D sediment transport model and a morphological model (for bottom update) (Figure 3). The model's methodological approach is described in Oliveira (2001) and DHI (2008). The wave transformation processes considered are shoaling, refraction, directional dispersion, and wave decay due to energy dissipation associated to bottom dissipation and wave breaking. The processes which contribute to the sediment transport induced by the waves which progress towards the coastline considered in the model are: the wave vertical and horizontal asymmetry, the Lagrangian flux, the circulation current next to the boundary layer (or streaming), the surface mass displacement due to breaking (or surface roller) and the undertow. Since infragravity waves (resultant from nonlinear harmonic interactions from short wave groups) are not taken into account, the model does not consider swash motions (which up to a large degree result from wave group forcing of infragravity waves) (Tucker, 1954), that is, the extension of the active zone ends in the last wet cell due to the combined action of the setup (a lower frequency rise in the water level due to wave breaking) and the short (or gravity) waves. The model resolves the two sediment transport modes, bed load and suspension. At the end of each time steep, the model updates the bottom through the application of the continuity equation to the sediments.

In this model the main calibration parameters, discussed with more detail in Oliveira $(2011 ; 2012)$, are the breaking parameters Gamma1 $\left(\gamma_{1}\right)$ and Gamma2 $\left(\gamma_{2}\right)$, the turbulence parameter Beta $(\beta)$, the maximum angle of submerged stable bottom (Max Angle) and the Scale Parameter ( $\left.\alpha_{\text {scale }}\right)$ (Table 1).

The dimensionless parameters $\gamma_{1}$ and $\gamma_{2}$ are considered for the

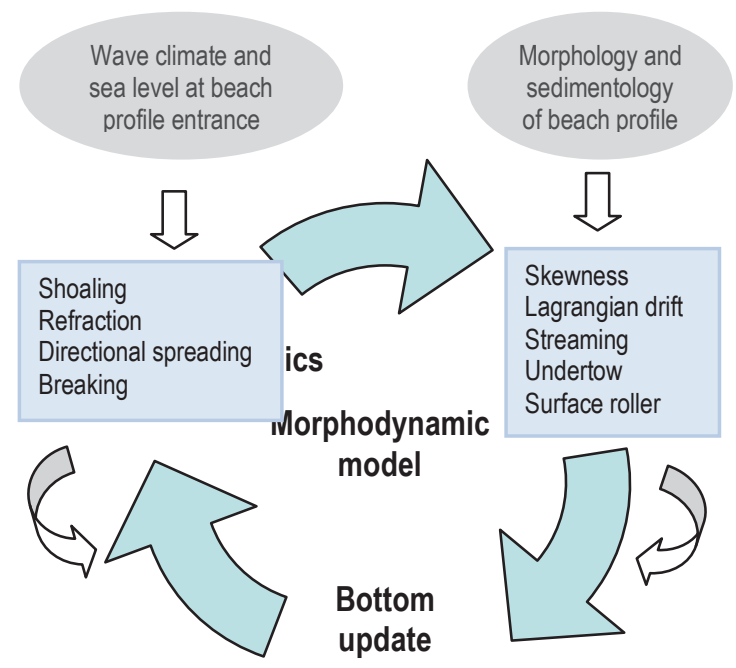

Figure 3. Simplified scheme of the numerical model. prediction of the maximum wave height, $H_{\max }$, necessary to estimate the energy dissipated according to Battjes and Janssen (1978). The two parameters are considered in the following formulation

$$
H_{\max }=\frac{\gamma_{1}}{\gamma_{2}} \tanh \left(\frac{\gamma_{2} k h}{\gamma_{1}}\right)
$$

where $k$ is the wave number and $h$ the depth. The breaking parameter $\gamma_{1}$ describes the maximum wave steepness, $H / L$. According to Battjes and Stive (1984), the breaking parameter $\gamma_{2}$ is calculated as

$$
\gamma_{2}=0.5+0.4 \tanh \left(33 s_{0}\right)
$$

where $s_{0}$ is the offshore wave steepness, equal to $H / L_{0}$, and $L_{0}$ is the offshore wave length.

The parameter $\beta$ is an empirical parameter that describes the relationship between the energy dissipation rate and the surface roller area, through the formulation proposed by Dally and Brown (1995)

$$
\frac{d}{d x}\left(E_{f} \cos \alpha\right)+\frac{d}{d x}\left(\frac{1}{2} \rho_{r} c^{2} \frac{A}{T} \cos \alpha\right)=\rho_{r} g \beta \frac{A}{T}
$$

where $E_{f}$ is the wave energy flux, $\alpha$ is the incident wave angle, $\rho_{r}$ is the density of the roller (including air bubbles), $A$ is the surface roller area, $T$ is the mean period and $g$ is the gravitational acceleration. The dimensionless parameter $\beta$ was defined based on laboratory experiments for regular waves (Dally and Brown, 1995). The recommended value is 0.15 , independently of the type of waves, regular or irregular, considered.

The parameter Max Angle is the maximum angle of the sea bottom before submerged avalanche occurs, that is, the maximum angle that the bottom slope can have before the bathymetry being modified due to slope failure.

The dimensional parameter $\alpha_{\text {scale }}$ is a diffusion coefficient to smooth out large gradients in the transport field. It is a parameter which reflects the cross shore exchange of momentum and is proportional to a characteristic length scale over which the transport is smoothed (DHI, 2008). It affects the shape of the developing bars and its increase causes longer bars.

To evaluate the numerical results, a statistical parameter suitable to the variable to analyze, which is depth, was calculated in addition to the visual observation of the similarity between predictions and measurements of the beach profile. Such statistical parameter was the Brier Skill Score (BSS) proposed by van Rijn et al. (2003), which compares profile predictions $\left(z_{b, c}\right)$ and measurements $\left(z_{b, m}\right)$ with the initial profile $\left(z_{b, 0}\right)$ and has into account the measuring error $\partial$ (here assumed as null). It can be defined has

$$
B S S=1-\frac{\left\langle\left(\left|z_{b, c}-z_{b, m}\right|-\partial\right)^{2}\right\rangle}{\left\langle\left(z_{b, 0}-z_{b, m}\right)^{2}\right\rangle}
$$

where the angular parenthesis denote average.

The classification for assessing the performance of morphological models proposed by van Rijn et al. (2003) can be seen in Table 2.

\section{RESULTS AND DISCUSSION}

\section{Numerical Modelling at the Reduced Scale}


Table 2. Classification proposed by van Rijn et al. (2003).

\begin{tabular}{lc}
\hline \hline Classsification & Brier Skill Score $($ BSS $)$ \\
\hline Excellent & $1.0-0.8$ \\
Good & $0.8-0.6$ \\
Reasonable/fair & $0.6-0.3$ \\
Poor & $0.3-0.0$ \\
Bad & $<0.0$ \\
\hline
\end{tabular}

The numerical model with the default parameters simulates erosion of the beach face, process which was not observed in the laboratory experiment, where the erosion phenomenon occurred localized in the profile zone located between 11-17 $\mathrm{m}$ distance from the origin of the abscissa of the profile Cartesian coordinate system. Consequently, the model simulates a profile retreat at the waterline level with approximately $1 \mathrm{~m}$ which was not observed in the laboratory (Figure 4).

Of the five calibration parameters tested, the one which caused the largest modifications to the numerical results obtained with the default parameters was the Gamma2. The change of Gamma2 from 0.6 to 0.4 was the calibration procedure which provided the best similarity between the numerical and the experimental results. The BSS changed from -0.74 to -0.25 (Table 3 ). It was concluded that the Gamma2 parameter was the most effective in the calibration of the numerical model at the reduced scale.

\section{Numerical Modelling at the Prototype Scale}

The numerical model with the default parameters simulates irrelevant modifications of the beach profile. Therefore, it does not simulate the erosion which occurred in the top of the submerged experimental profile (Figure 4).

Like for the case of application of the numerical model at the reduced scale, the calibration parameter which caused the greatest modifications of the beach profile relatively to the numerical results with the default parameters was the Gamma2. However, for all the Gamma2 values tested, with the exception of the default value, 0.6, the Gamma2 parameter caused erosion in the zone of the beach face (like in the results of the simulations performed at the reduced scale). Based on the error indicator BSS, it was the calibration parameter Scale Parameter with the value 0.8 which provided the best profile result at the prototype scale (Table 4).

\section{Comparison between Models at both Scales}

The application of the numerical model with the default parameters at both scales showed that the model was only able of

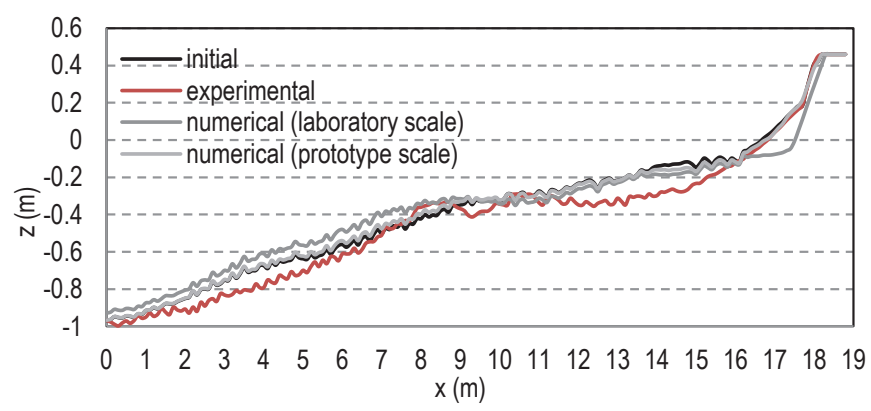

Figure 4. Profile evolution after 11 hours. Numerical results with the default parameters (at laboratory and prototype scales).
Table 3. BSS for the tests at the reduced scale.

\begin{tabular}{lc}
\hline \hline Test & Brier Skill Score $($ BSS $)$ \\
\hline Default parameters & -0.745 \\
Gamma1 $=0.6$ & -0.529 \\
Gamma1 $=0.7$ & -0.495 \\
Gamma1 $=0.8$ & -0.592 \\
Gamma1 $=0.9$ & -0.667 \\
Gamma1 $=1$ & -0.589 \\
Gamma2 $=0$ & -2.186 \\
Gamma2 $=0.4$ & -0.252 \\
Gamma2 $=0.8$ & -2.096 \\
Gamma2 $=1$ & -5.049 \\
Beta $=0.1$ & -0.803 \\
Beta $=0.2$ & -0.747 \\
Max Angle $=15$ & -0.479 \\
Max Angle $=20$ & -0.532 \\
Scale Parameter $=0.8$ & -0.712 \\
Scale Parameter $=1.2$ & -0.845 \\
\hline
\end{tabular}

the erosion observed in the laboratory (which was localised in the top of the submerged part of the profile) (Figure 4). The error indicator BSS highlights a better performance of the numerical model with the default parameters at the prototype scale than at the reduced scale (Tables 3 and 4). Nevertheless, based on the van Rijn et al. (2003) classification (Table 2), it should be pointed out that the result (performance) is poor in the first case and bad in the second case, in which the profile erosion process is underestimated and overestimated, respectively. When considering these results, it is important to bare in mind that the sediment escaped into the flume platform (out of the monitoring zone) during the laboratory experiment must have given a large contribution (not possible to quantify) to the numerical and experimental disagreement in the lower part of the submerged profile, making the numerical model performance worse than it really was.

The numerical results obtained at both scales, prototype and reduced scales, were compared for each of the best cases for each calibration parameter (Figure $5 \mathrm{a}-\mathrm{e}$ ). The evaluation of each of the best cases was based in the simultaneous analysis of the similarity between the numerical and experimental profiles and the error indicator BSS. It was observed that, with the exception of the calibration parameter Scale Parameter, for which the best value obtained was 0.8 , none of the calibration parameters had coincident value for the best case at both scales. For the reduced scale and prototype scale, respectively, were obtained the following values for the best cases: 1.0 and 0.8 for the parameter

Table 4. BSS for the tests at the prototype scale.

\begin{tabular}{lc}
\hline Test & Brier Skill Score (BSS) \\
\hline Default parameters & 0.043 \\
Gamma1 $=0.6$ & 0.008 \\
Gamma1 $=0.7$ & 0.035 \\
Gamma1 $=0.8$ & 0.049 \\
Gamma1 $=0.9$ & 0.045 \\
Gamma1 $=1.0$ & 0.048 \\
Gamma2 $=0.0$ & -0.204 \\
Gamma2 $=0.8$ & -0.184 \\
Gamma2 $=1.0$ & -1.182 \\
Beta $=0.1$ & 0.048 \\
Beta $=0.2$ & 0.042 \\
Max Angle=15 & 0.008 \\
Max Angle $=20$ & 0.033 \\
Scale Parameter $=0.8$ & 0.054 \\
Scale Parameter $=12$ & 0.033
\end{tabular}


a)

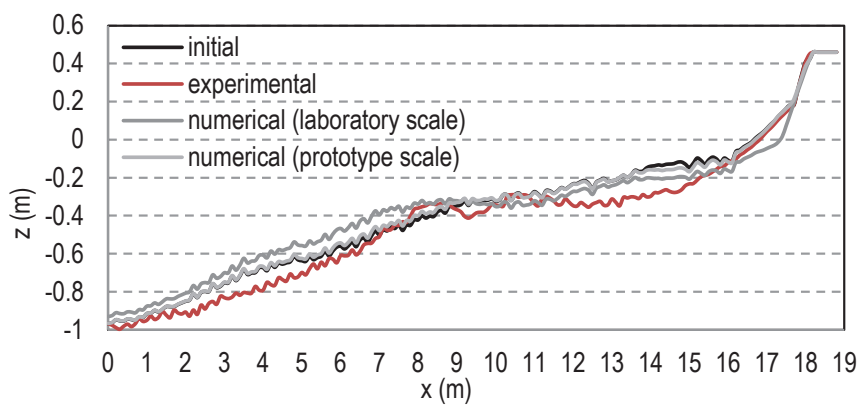

c)

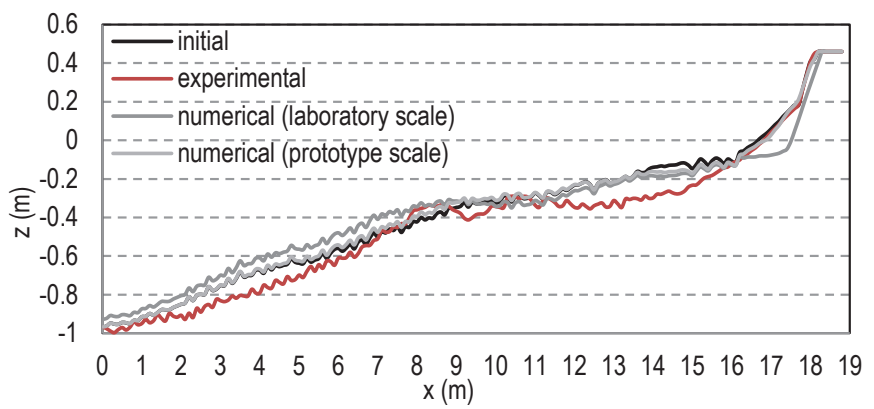

b)

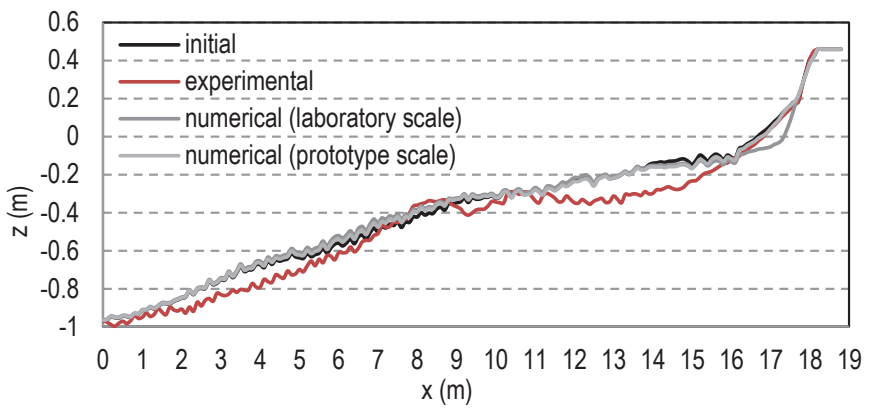

d)

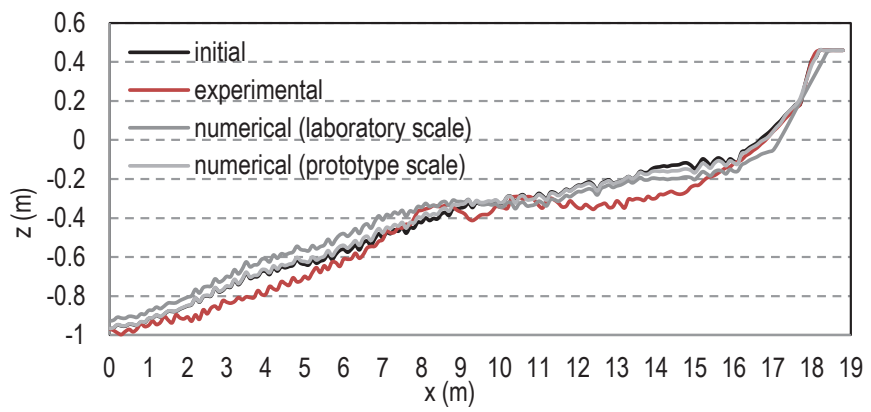

e)

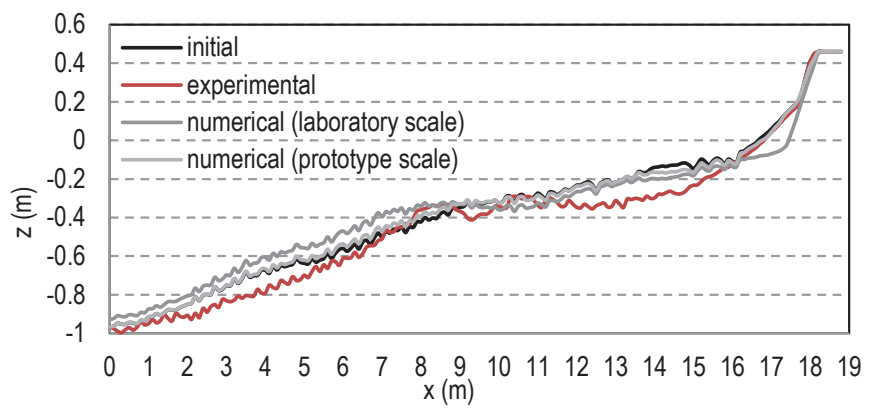

Figure 5. Profile evolution after 11 hours. Numerical results for each calibration parameter best case (at laboratory and prototype scales): a) Gamma1, b) Gamma2, c) Beta, d) Max Angle and e) Scale Parameter.

Gamma1; 0.4 and 0.6 (the default value) for the parameter Gamma2; 0.15 (the default value) and 0.1 for the parameter Beta; and $20^{\circ}$ and $30^{\circ}$ (the default value) for the parameter Max Angle. It was also observed that the calibration parameter which provided the best results at each scale was not the same: at the reduced scale was the parameter Gamma2 (for BSS equal to -0.25); and at the prototype scale was the Scale Parameter (for BSS equal to 0.05). These results steer to conclude that the calibration of the numerical model depends on the scale of its application.

Like for the case of application of the numerical model with the default parameters, the error indicator BSS indicates a better performance of the numerical model calibrated at the prototype scale than at the reduced scale (Tables 3 and 4). Nevertheless, it remains the fact that the profile erosion process is overestimated at the reduced scale and underestimated at the prototype scale.

\section{CONCLUSIONS}

The study aimed to validate a morphodynamic numerical model at different scales (reduced model scale and prototype scale) and ascertain on the existence of scale effects in the numerical modelling. To achieve this goal laboratory experiments at reduced scale were performed in an irregular wave flume to simulate a case of beach profile erosion. The case study was then reproduced numerically at the reduced laboratory scale and at the prototype scale. In a first phase, the results of both applications were validated against the laboratory results (upscaled for the prototype scale validation). In a second phase, the numerical results at the prototype scale, after downscaled to the laboratory scale, were compared with the numerical results at the laboratory scale. The evaluation of the numerical model performance was based on the observation of the similarity between predictions (numerical results) and measurements (laboratory results) and on the error indicator BSS, which was calculated for each test. In the applications of the numerical model, at both scales, tests with the default parameters recommended by the model authors were performed before the calibration.

It was concluded that the beach profile erosion process was overestimated at the reduced scale and underestimated at the prototype scale in both model applications, that is, with the default 
parameters and in the calibration. The indicator error BSS highlights a better performance of the numerical model at the prototype scale than at the reduced scale, despite, based on the classification proposed by van Rijn et al. (2003), in the first case the performance is poor and in the second case is bad. When evaluating the model's performance it should be accounted that the sediment escaped into the flume platform (out of the monitoring zone) during the laboratory experiment must have given a large contribution to the numerical and experimental disagreement in the lower part of the submerged profile, causing, thus, an apparent worse performance of the numerical model than it really was.

It was also concluded that the most influent calibration parameter in the beach profile modification at both scales was the Gamma2 parameter, but that only in the case of numerical modelling at the reduced scale this was the most effective calibration parameter. In the case of numerical modelling at the prototype scale, the Scale Parameter was the most effective calibration parameter.

From the calibration process at both scales, it was also concluded that, with the exception of the calibration parameter Scale Parameter, none of the other calibration parameters had coincident values in both scales.

This study led to the final conclusion that there are scale effects in the numerical modelling of beach profile erosion with the Litprof model.

\section{ACKNOWLEDGEMENT}

This study was executed within the scope of the MorFeed project (PTDC/AAC-AMB/100092/2008), financed by Fundação para a Ciência e a Tecnologia (FCT), the Portuguese foundation for science and technology.

\section{LITERATURE CITED}

Battjes, J.A. and Stive, M.J.F., 1984. Calibration and verification of a dissipation model for random breaking waves. Proceedings of the 19th Conference on Coastal Engineering (Houston, Texas, USA), pp. 649 660 .

Battjes, J.A. and Janssen, J.P.F.M., 1978. Energy Loss and Set-Up due to Breaking of Random Waves. Proceedings of the 16th Int. Conference on Coastal Engineering (Hamburg, Germany), ASCE, pp. 569-587.

Dally, W.R. and Brown, C.A., 1995. A modeling investigation of the breaking wave roller with application to cross-shore currents. Journal of Geophysical Research, 100, C12, 24. 873-24.883.

DHI, 2008. Profile development. LITPROF user guide. Danish Hydraulic Institute, $74 \mathrm{pp}$.

Freire, P., Oliveira, F.S.B.F., Capitão, R., Fortes, C. and Costa, M., 2004. Cross-shore evolution of Buarcos beach, Portugal. Proceedings of the 29th Int. Conference on Coastal Engineering (Lisboa, Portugal), ASCE, pp. 2314-2326.

Freire, P., Sancho, F. and Oliveira, F.S.B.F., 2008. Composite modelling of sediment dynamics for propagating waves reaching coastal defences. Second International Conference on the Application of Physical Modelling to Port and Coastal Protection (Coastlab08), (Bari, Italy), pp. 87-90.

Frostick, L.E, McLelland, S.J. and Mercer, T.G., 2011. User guide to physical modelling and experimentation: Experience of the HYDRALAB Network. CRC Press/Balkema, Leiden, The Netherlands, 245 pp.

Larangeiro, S.H.C.D., Oliveira, F.S.B.F. and Freire, P.M.S., 2003 Longshore sediment transport along a sandy coast with hard rock outcrops. Shore and Beach, 71, 2, 20-24.

Oliveira, F.S.B.F., 2001. Cross-shore littoral transport. Report 1 Mathematical modelling of the hydrodynamics and sediment transport in coastal zones. Laboratório Nacional de Engenharia Civil, Lisboa, Portugal, $39 \mathrm{pp}$.

Oliveira, F.S.B.F., 2002. Effect of the sea level variation in the offshore limit of the surf zone of Buarcos, Portugal. Littoral 2002, The changing coast. EUROCOAST/EUCC (Porto, Portugal), pp. 363-368.
Oliveira, F.S.B.F., 2011. Evaluation of the beach and dune erosion model XBeach: preliminary results. $11^{\text {th }}$ Water Congress (Porto, Portugal), CD-ROM, $18 \mathrm{pp}$

Oliveira, F.S.B.F., 2012. Dune erosion with the XBeach and Litprof models. Journal of Integrated Coastal Zone Management 12, 2, 195 222.

Tucker, M.J., 1954. Surfbeats: sea waves of 1 to 5 minutes' period. Proceedings Royal Society London, Ser. A 202, 565-573.

Van Rijn, L.C., Walstra, D.J.R., Grasmeijer, B., Sutherland, J., Pan, S. and Sierra, J.P., 2003. The predictability of cross-shore bed evolution of sandy beaches at the time scale of storms and seasons using processbased profile models. Coastal Engineering, 47, 295-327. 